\title{
Material Differentiation by Differential Computed Tomography
}

\author{
Josef Prokop*, Libor Sveda, Alexandr Jancarek and Ladislav Pina \\ Czech Technical University in Prague, Faculty of Nuclear Sciences and Physical Engineering, \\ Brehova 7, 11519 Prague, Czech Republic
}

\begin{abstract}
This paper presents the study on the feasibility of a differentiation of the individual materials using so called dual energy computed tomography. The individual pixels on tomogram are the results of X-ray attenuation penetrating through the sample from different angles of projections. Attenuation is caused by absorption and scattering of radiation. The contribution of these two main mechanisms is dependent on atomic number, electron density, and also the energy of the X-ray photons. The material characteristics may be quantitatively analyzed when the composition in to atomic number or density information is carried out. Therefore, two different X-ray spectra were used by application of filters. The principle of proposed method results in a numerical approach with the associated detector calibration. We tested first the method on the samples of known composition. From the results it was shown that we are able to obtain removal of "beam hardening" and the estimation of material composition. For further more precise determination of materials, the proposed method will be used with the respect to detector acquisition improvement. [doi:10.2320/matertrans.I-M2011851]
\end{abstract}

(Received November 13, 2009; Accepted October 29, 2011; Published January 25, 2012)

Keywords: X-ray, computed tomography, dual energy, spectrum, material differentiation

\section{Introduction}

Conventional X-ray imaging methods in tomographic mode, providing information about the examined object do not characterize it sufficiently. The image interpretation for multicomponent specimens is represented by the differences in a matrix of attenuation coefficients. These differences can be due to differences in the electron density and the atomic number, or their combination. In contrast, differential tomography, referred to as dual energy X-ray method, allows the decomposition of CT image into density and effective atomic number information. The decomposition is performed with the aid of knowledge of physics of X-ray interaction with matter and necessary mathematical apparatus.

\section{X-ray Interaction with Matter}

The attenuation of the X-ray with matter is the result of interaction mechanisms, dominated by the photoeffect absorption and Compton scattering. The photoeffect absorption is strongly dependent on the atomic number and density of the specimen being examined. The Compton scattering is a function of the electron density of the material. The linear attenuation coefficient can be expressed as ${ }^{1)}$

$$
\mu(E, \rho, Z)=\mu_{\mathrm{m}}(E) \times \rho=\rho\left[\mathrm{a}(E)+\mathrm{b}(E) \times Z^{k} / E^{l}\right]
$$

where $\mu$ is the linear attenuation coefficient, $\rho$ is the material density, $\mu_{\mathrm{m}}$ is the mass attenuation coefficient, $Z$ is the atomic number. The $k$ values typically lie between 3 and 4 ; and $l$ values between 3 and 3.5. For energies less than $150 \mathrm{kV}$, the Compton scattering is not dependent on energy, $E$.

\section{Dual Energy Method}

Differential tomography, referred to as dual energy X-ray method, consists in processing of at least two tomographic images acquired at two or more various energy levels. This system can be easily solved for monochromatic energy

*Graduate Student, Czech Technical University in Prague spectra and ideal detectors, but become complex when considering realistic spectra, detector sensitivity, and system non-linearity. ${ }^{2-4)}$

Using filters, two different X-ray spectra were applied. In our study we considered the available filter elements with the K-edge discontinuity from the range of 40 to $100 \mathrm{keV}$ since the applied voltage was at that defined range. The thickness of filters: $\mathrm{Gd}, \mathrm{Sb}, \mathrm{Yb}, \mathrm{Au}, \mathrm{Pt}, \mathrm{Cu}, \mathrm{Ho}, \mathrm{Pb}$ varied from 100 to $250 \mu \mathrm{m}$. The used filters with appropriate thickness produces a method to quasi-monochromatize X-ray beams. Figure 1 shows the application of Gd filter on X-ray spectrum. Due to this filtration we optimize the X-ray energy ranges.

As one approach, the system of two integral equations may be set and analytically solved. Since the analytical way is considerably demand on the knowledge of detector efficient and requires the intensive computation, in this study we focused on numerical approach based on the function $F(Z)$ which is monotonic with respect to atomic number. This function as the portion of two linear attenuation coefficients is only dependent on $Z$.

$$
k=\mu_{1} / \mu_{2}=F(Z) \Rightarrow Z=F^{-1}\left(\mu_{1} / u_{2}\right)
$$

Before plotting the function, the calibration was carried out for the specific combination of a source, detector, and filters. The ideal function should be derived from as most as possible elements tested. Considering the elements nature and elements availability, the fitness by cubic polynomial function was applied to set up the value for not tested samples.

\section{Results}

The measurement was carried out on our developed CT scanner. Figure 2 shows the basic components of arrangement for the experiment. Different combination of filters was applied to our experiment. As the result of the tomographic reconstruction for different samples, $\mathrm{Pb}$ and $\mathrm{Yb}$ filters provide the best combination for determination of atomic numbers of elements in $Z$ between 20 and 50. For this combination of filters plotting $k$ against $Z$ has the monotonic characteristic. 


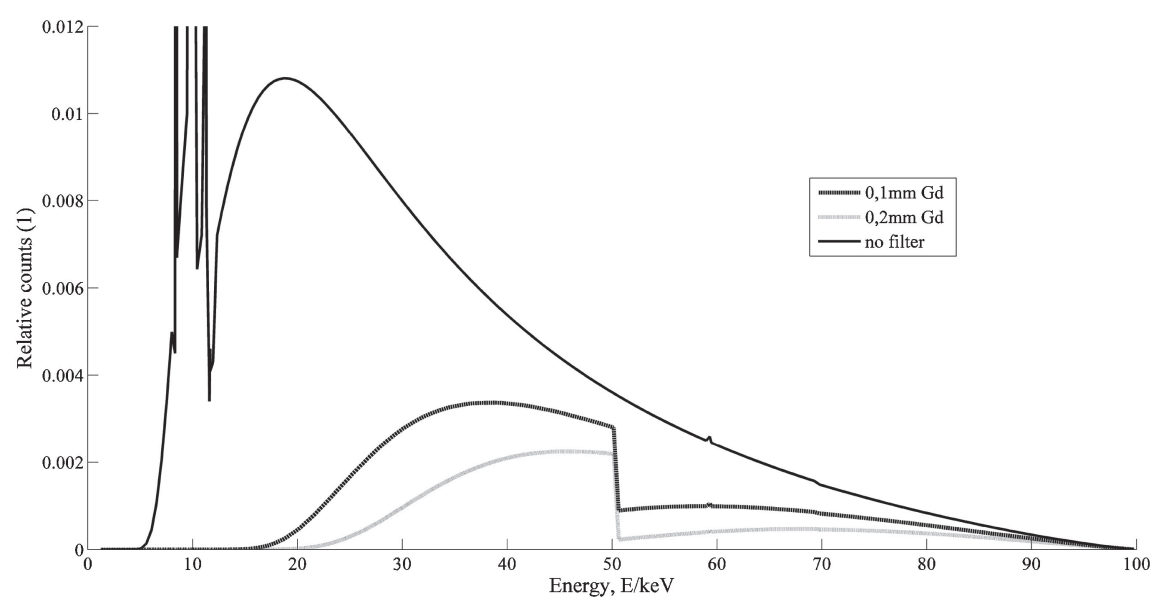

Fig. 1 Gd filter influence on the $100 \mathrm{kV}$ X-ray spectrum.

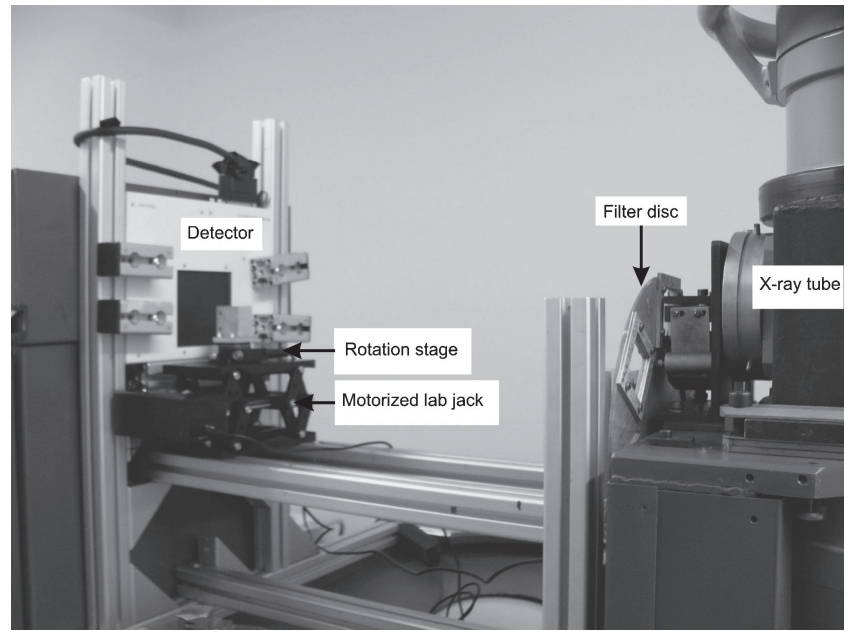

Fig. 2 In-house X-ray computed tomograph. (a)

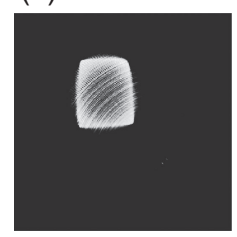

(b)

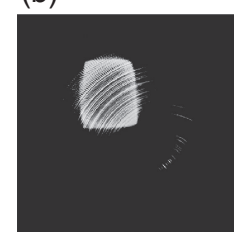

(c)

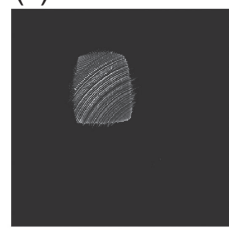

Fig. 3 Interpretation of visualization of $Z$ decomposition. (a) tomogram of Mo sample obtained through $\mathrm{Pb}$ filter, (b) tomogram of Mo sample obtained through $\mathrm{Yb}$ filter, (c) image of atomic number information after decomposition.
In Fig. 3, the visual interpretation of application of dual energy method is shown. The tomograms obtained by the application of $\mathrm{Pb}$ and $\mathrm{Yb}$ filters are further decomposited into image providing information about $Z$. In this illustration, the sample from molybdenum was proceeded to this procedure. The ring artifacts in the images are due to a detector fault.

\section{Conclusion}

The results show a rough estimation of the material identification and removal of beam hardening. We encounter the difficulties with small dynamic range and imperfections of detector. The following step will require to compound the images with different exposure and/or develop the monochromator based on multilayers or crystals (Bragg reflection).

\section{Acknowledgements}

This work was supported by the grant "Multiscale modelling and X-ray tomography", grant No. 105/09/ 1830, grant No. FR-TI1/139 and by the CTU grant No. CTU0916414.

\section{REFERENCES}

1) C. Rizescu, C. Besliu and A. Jipa: Nucl. Instrum. Methods Phys. Res. A 465 (2001) 584-599.

2) B. J. Heismann, J. Leppert and K. Stierstorfer: J. Appl. Phys. 94 (2003) 2073-2079.

3) M. Van Geet, R. Swennen and M. Wevers: Sediment. Geol. 132 (2000) 25-36.

4) R. T. Lopes, E. B. Costa and E. F. O. de Jesus: Appl. Radiat. Isot. 53 (2000) 665-671. 